\title{
Unusual Development of Pulmonary Tumor Embolism from Controlled Liver Metastases of Transitional Cell Carcinoma: An Autopsy Case
}

\author{
Hiromitsu Kitayama ${ }^{1}$, Taro Yokota ${ }^{1}$, Tomohiro Kondo ${ }^{1}$, Junko Sugiyama ${ }^{1}$, \\ Michiaki Hirayama ${ }^{2}$, Yumiko Oyamada ${ }^{3}$ and Yasushi Tsuji ${ }^{1}$
}

\begin{abstract}
Clinicians generally suspect pulmonary tumor embolism (PTE) with uncontrolled carcinomas which often spread to lungs. We, however, experienced an autopsy case of diffuse microscopic PTE despite controlled liver metastases of transitional cell carcinoma (TCC). A 66-year-old man with progressing respiratory symptoms showed almost normal chest findings on computed tomography. Although liver metastases were successfully shrunk by chemotherapy, the patient died from aggressive respiratory failure. An autopsy revealed small pulmonary vessels showing diffuse tumor emboli. TCC can cause PTE even if liver metastases are controlled. We must therefore be aware that PTE can manifest as respiratory symptoms without any computed tomography findings.
\end{abstract}

Key words: pulmonary tumor embolism (PTE), liver metastases, transitional cell carcinoma (TCC), bladder cancer, respiratory failure, lymphangitic carcinomatosis

(Intern Med 55: 2453-2456, 2016)

(DOI: 10.2169/internalmedicine.55.6662)

\section{Introduction}

Pulmonary tumor embolism (PTE) is an uncommon form of lung metastases and causes dyspnea in cancer patients. The occlusion of the pulmonary microvasculature by tumor cells and associated thrombi can present with a subacute and progressive clinical picture that resembles thromboembolic disease (1).

Clinicians can easily recognize the risk of PTE from primary carcinomas of stomach, liver, kidney, breast, and prostate $(2,3)$, especially when they are not well controlled. However, the rare PTE from transitional cell carcinoma (TCC) is infrequently diagnosed due to the difficulty of diagnosis before the patient's death (4). Furthermore, PTE develops even more rarely if liver metastases are well controlled. We herein report an autopsy case of diffuse microscopic PTE which developed from controlled liver metastases of TCC. The characteristic symptom was respiratory fail- ure with almost normal chest computed tomography (CT) findings.

\section{Case Report}

The patient was a 66-year-old Japanese man with recurrent bladder cancer. He had a 20-pack-year history of smoking. First, he underwent standard systemic chemotherapy with gemcitabine and cisplatin for recurrent pelvic lymph node metastases, which resulted in complete remission. However, two years later, the patient developed liver metastases and received several regimens of chemotherapy with gradually worsening and ultimately no response. We chose hepatic artery injection with epirubicin as salvage chemotherapy. During seven months of this therapy, the levels of surrogate tumor markers, carcinoembryonic antigen and carbohydrate antigen 19-9, were well controlled, and CT scans showed shrinkage of the liver metastases (Fig. 1a and b). However, the patient developed a gradually progressing dry

${ }^{1}$ Department of Medical Oncology, Tonan Hospital, Japan, ${ }^{2}$ Department of Gastroenterology, Tonan Hospital, Japan and ${ }^{3}$ Department of Surgical Pathology, Tonan Hospital, Japan

Received for publication October 13, 2015; Accepted for publication December 8, 2015

Correspondence to Dr. Hiromitsu Kitayama, m02032hk@jichi.ac.jp 

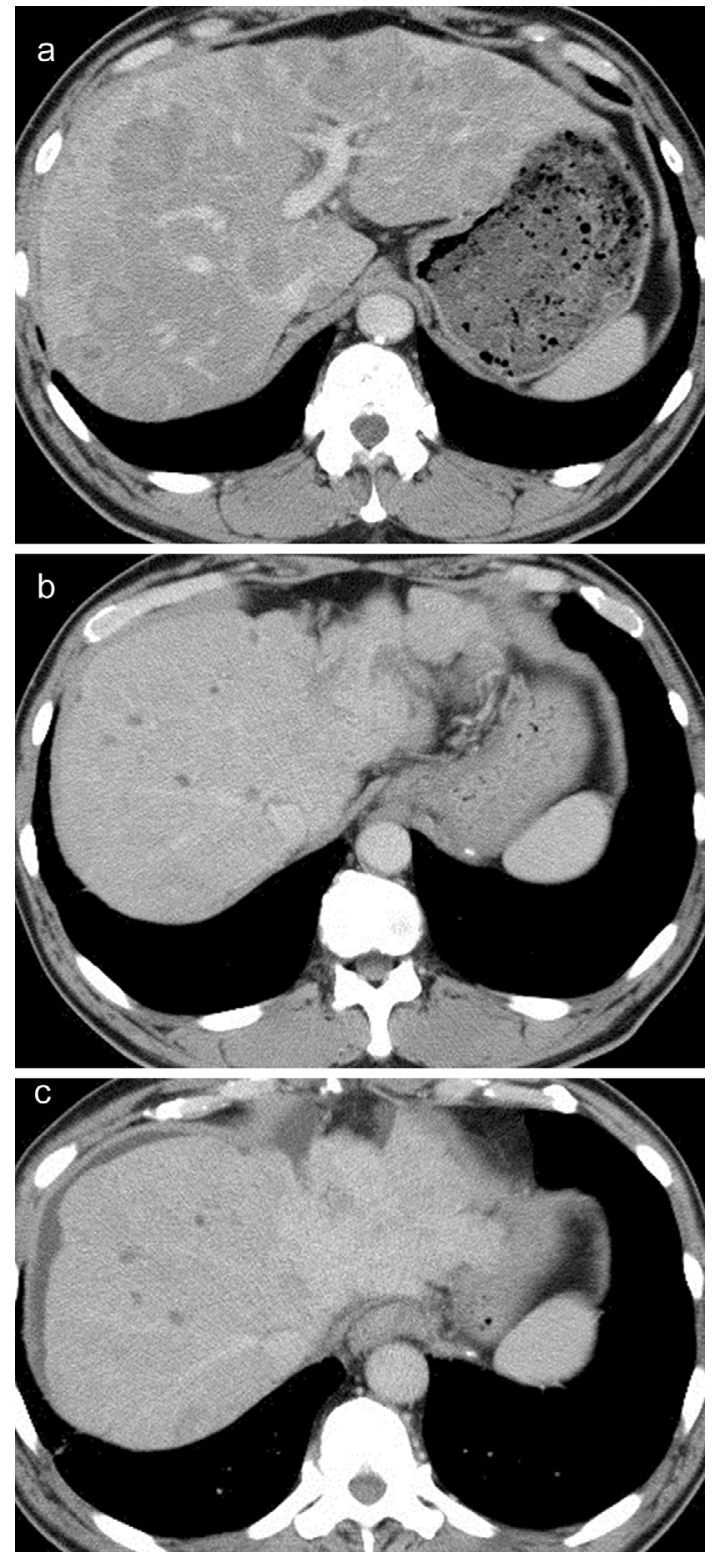

Figure 1. Computed tomography before (a) and seven months after (b) hepatic artery injection of epirubicin. At the time the patient's respiratory symptoms worsened, (c) a shrinkage of liver metastases was observed.

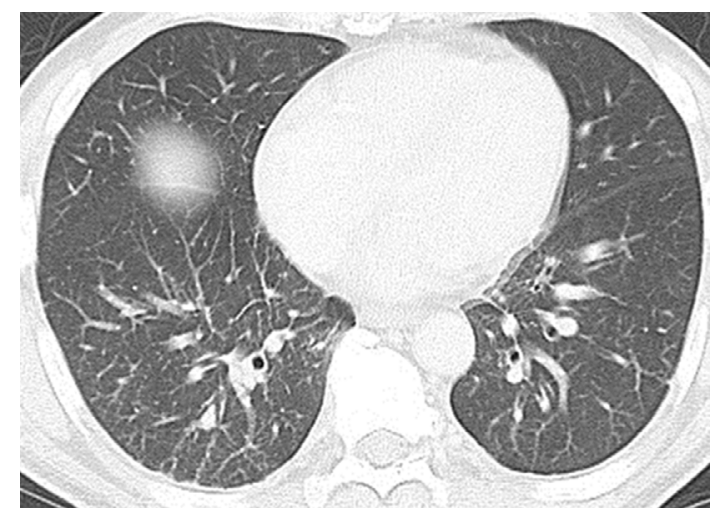

Figure 3. Computed tomography showed only slight thickening of the peribronchovascular interstitium and subpleural interlobular septum, and multiple tiny nodules in the right lower lobe of lungs.

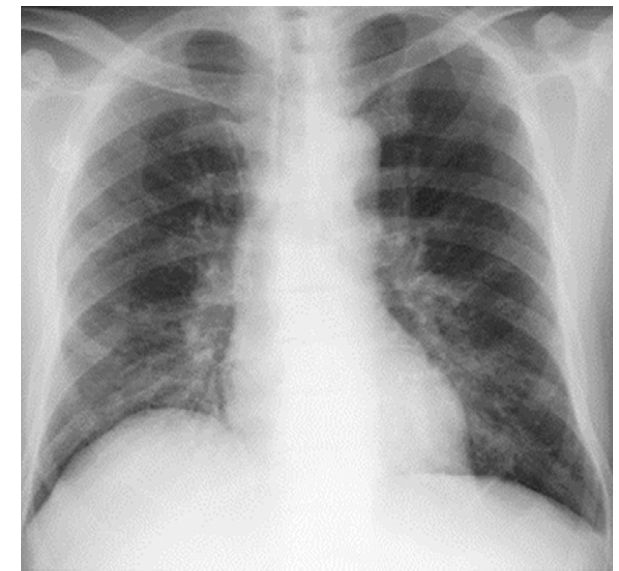

Figure 2. Chest radiography showed only slight groundglass opacities and trabecular shadows.

cough and shortness of breath despite normal chest CT findings. His respiratory symptoms rapidly worsened, and he was admitted due to severe respiratory failure. His platelet count was not decreased, partial thromboplastin time and activated partial thromboplastin time were not prolonged, and the plasma D-dimer level was $27 \mu \mathrm{g} / \mathrm{mL}$. Chest radiography showed only slight ground-glass opacities and trabecular shadows (Fig. 2). Chest CT scans showed only slight thickening of the peribronchovascular interstitium and subpleural interlobular septum, and multiple tiny nodules in the right lower lobe (Fig. 3) with no pulmonary embolism. A pulmonary function test showed moderate impairment of the diffusion capacity with no obstructive or restrictive impairment. Echocardiography showed no evidence of heart failure or pulmonary hypertension. CT scans also showed no apparent progression of the liver metastases (Fig. 1c) and lymphadenopathy. Ventilation-perfusion scanning and pulmonary artery cytology were impossible to perform due to severe hypoxia. The patient received steroid pulse therapy, however, respiratory failure progressed rapidly without any response, and he died after ten days from this admission.

An autopsy revealed bladder cancer with diffuse liver metastases (Fig. 4) and mild lymphangitic carcinomatosis with multiple lymph node metastases in the chest and abdomen. According to these specimens, the patient was diagnosed with TCC with grade of differentiation G3. Lymphangitic carcinomatosis existed in the peribronchovascular interstitium and subpleural interlobular septum with pulmonary intestinal edema. Small pulmonary vessels showed diffuse tumor emboli (Fig. 5) without dilation of the capillary. This case differed from pulmonary tumor thrombotic microangiopathy, a specific type of PTE, due to the absence of fibrocellular or fibromuscular intimal proliferation and thrombi in the vessels. Extensive infiltrations of the blood vessels were also observed in other organs.

\section{Discussion}

According to the findings associated with this case, we arrived at two important clinical suggestions. First, TCC can 


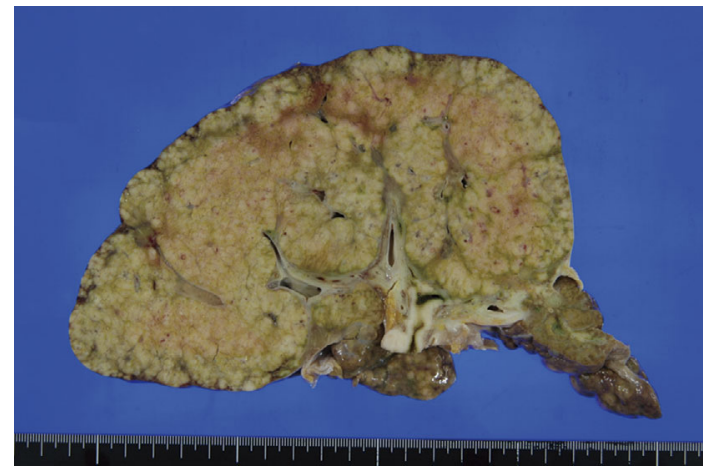

Figure 4. Macroscopic findings revealed a rapid increase in the growth of diffuse liver metastases.

cause PTE even if liver metastases are well controlled. This was a rare case of diffuse microscopic PTE from TCC without pulmonary hypertension. Autopsy series estimate that the incidence of PTE is between 3\% and 26\% among patients with solid tumors $(2,5)$, however, the histological type is mostly adenocarcinoma including breast, lung, and gastric cancer (1). TCC was reported in only 2 of 109 cases (2\%) of PTE (1). PTE causes serious pulmonary disease in less than $1 \%$ of all patients with cancer (3). In many cases, patients may develop liver failure before symptomatic PTE, especially with primary lesion anatomically distant from the chest or liver, such as bladder cancer. To the best of our knowledge, symptomatic microscopic PTE from TCC has been reported in 11 cases, and only 5 cases presented without pulmonary hypertension (Table) (6-14). However, we should pay attention to new lung metastases in patients receiving long-term chemotherapy for liver metastases. Metastatic cancer to the liver with hepatic venous invasion is considered to be the most common cause of PTE, and PTE from TCC with liver metastases was reported in 4 of 11 cases (Table). In the present case, the diffuse liver metastases may have invaded and caused PTE through the inferior vena cava. The explanation for how PTE caused respiratory symptoms despite the patient suffering from TCC was likely effective local chemotherapy by hepatic artery injection for liver metastases. The relatively long control of liver metastases might lead to a metastatic variant resistant to chemotherapy that is able to infiltrate the blood vessels. Although the growth of diffuse liver metastases rapidly increased in the late stage of the patient's disease, it was well controlled by local chemotherapy at the time he developed the severe respiratory symptoms of PTE.

The second clinical suggestion that the case supports is that respiratory symptoms in metastatic cancer patients with almost normal chest CT findings can be diffuse microscopic PTE. In cancer patients with respiratory symptoms, clinicians must certainly first consider common diagnoses such as infection, thromboembolism, side effects of toxic chemotherapeutic regimens, and other pulmonary manifestations of the primary neoplasm. Of the many causes of respiratory symptoms in cancer patients, PTE is one of the most difficult to diagnose, particularly before death. In the first pub-

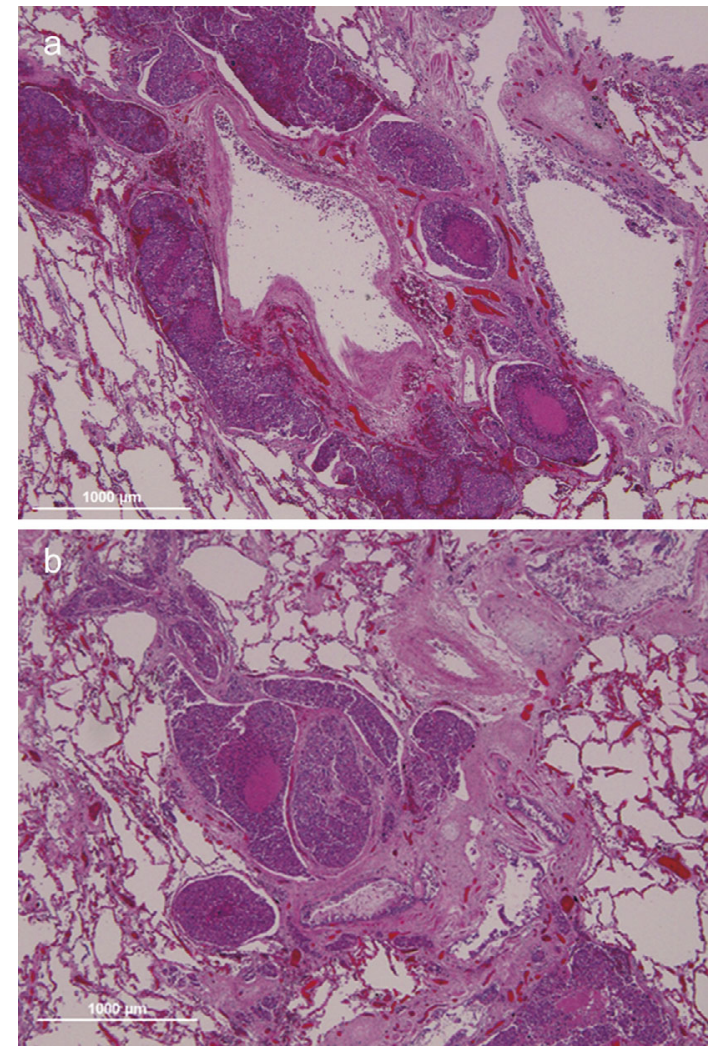

Figure 5. Microscopic findings revealed a pulmonary venule (a) and arteriole (b) showing diffuse tumor emboli (Hematoxylin and Eosin staining).

lished autopsy series, Kane et al. noted that only 1 of 8 patients had a parenchymal abnormality on plain radiography (2). Some investigators, in an attempt to distinguish carcinomatosis and tumor emboli, suggested that lymphatic disease is more likely to have an interstitial infiltrate on chest radiography or CT (15). Slight thickening of the peribronchovascular interstitium and subpleural interlobular septum was observed in this case, however, nearly all patients with PTE have associated lymphangitic carcinomatosis of varying degrees (16). Hypoxemia with a normal chest radiograph is the typical finding in a patient with tumor or thrombus emboli, whereas in the case of infection, interstitial fibrosis or lymphatic spread, the plain film may be sufficient for the diagnosis (1). Echocardiography may show pulmonary hypertension, which was reported in only $15 \%$ to $20 \%$ of patients with PTE (17) and 6 of 11 cases of PTE from TCC (Table). Thus, the severity of symptoms may not correlate with the degree of vascular obstruction (2). Ventilation-perfusion scanning and right heart catheterization including pulmonary artery cytology have a good diagnostic utility to distinguish microscopic pulmonary thromboembolism $(1,18)$. However, the availability of these diagnostic procedures is limited. Only 2 of 11 patients received appropriate therapy such as chemotherapy without unnecessary anticoagulation that led to a recovery (Table). Our patient's lymphangitic carcinomatosis was too mild to explain respiratory failure, and he had liver metastases and no obvious pulmonary thromboembolism. These findings suggest that he had diffuse micro- 
Table. Reported Diffuse Microscopic Pulmonary Tumor Embolism from Transitional Cell Carcinoma.

\begin{tabular}{llllllll}
\hline Case & $\begin{array}{l}\text { Age } \\
\text { (years) }\end{array}$ & Gender & $\begin{array}{l}\text { Pulmonary } \\
\text { hypertension }\end{array}$ & $\begin{array}{l}\text { Liver } \\
\text { metastasis }\end{array}$ & Therapy & Outcome & Reference \\
\hline 1. & 65 & Male & Present & Absent & Chemotherapy & Recovery & 6 \\
2. & 81 & Male & Absent & Absent & Antibiotics & Death & 6 \\
3. & 63 & Male & Absent & Present & BSC* & Death & 6 \\
4. & 71 & Male & Absent & Present & BSC & Death & 7 \\
5. & 86 & Male & Present & Present & BSC & Death & 8 \\
6. & 61 & Male & Present & Absent & Thrombolysis & Death & 9 \\
7. & 84 & Male & Present & Present & BSC & Death & 10 \\
8. & 70 & Male & Absent & Absent & Anticoagulation & Death & 11 \\
9. & 64 & Male & Absent & Absent & Chemotherapy & Recovery & 12 \\
10. & 70 & Male & Present & Absent & BSC & Death & 13 \\
11. & 77 & Male & Present & Absent & Anticoagulation & Death & 14 \\
\hline 12. & 66 & Male & Absent & Present & Steroids & Death & This case \\
\hline
\end{tabular}

* best supportive care

scopic PTE.

In conclusion, TCC can cause PTE even if liver metastases are well controlled, and respiratory symptoms in metastatic cancer patients with almost normal chest CT findings can be diffuse microscopic PTE. We must be aware that PTE can manifest as respiratory symptoms in cancer patients without any CT findings or pulmonary hypertension, particularly with liver metastases even from TCC. Diffuse microscopic PTE is often misdiagnosed and thus there may be many more "hidden" cases. Ventilation-perfusion scanning and right heart catheterization should be more readily used in such cases, where these tests may contribute to the diagnosis of "hidden" PTE, so the patients can receive more appropriate therapy, such as systemic chemotherapy without any unnecessary anticoagulation therapy.

\section{Author's disclosure of potential Conflicts of Interest (COI).}

Yasushi Tsuji: Honoraria, Ono Pharmaceutical.

\section{Acknowledgement}

The authors would like to thank the patient and patient's family for their kind co-operation, and David Hochman for reviewing the language of our article.

\section{References}

1. Roberts KE, Hamele-Bena D, Saqi A, Stein CA, Cole RP. Pulmonary tumor embolism: a review of the literature. Am J Med 115 : 228-232, 2003

2. Kane RD, Hawkins HK, Miller JA, Noce PS. Microscopic pulmonary tumor emboli associated with dyspnea. Cancer 36: 1473 1482, 1975

3. Winterbauer RH, Elfenbein IB, Ball WC Jr. Incidence and clinical significance of tumor embolization to the lungs. Am J Med 45: 271-290, 1968

4. Goldman SM, Fajardo AA, Naraval RC, Madewell JE. Metastatic transitional cell carcinoma from the bladder: radiographic manifestions. AJR Am J Roentgenol 132: 419-425, 1979.

5. Shields DJ, Edwards WD. Pulmonary hypertension attributable to neoplastic emboli: an autopsy study of 20 cases and a review of the literature. Cardiovasc Pathol 1: 279-287, 1992.

6. Fitzpatrick TM, Covelli HD, Tenholder MF. The acute and insidious onset of pulmonary metastatic transitional cell carcinoma
Chest 99: 498-500, 1991.

7. Arisawa C, Fujii Y, Higashi Y, Owada F, Shimizu S, Kaneko K. Acute respiratory failure resulting from diffuse microscopic pulmonary tumor emboli by bladder cancer: a case diagnosed at autopsy. Hinyokika Kiyo (Acta Urologica Japonica) 39: 475-478, 1993 (in Japanese, Abstract in English).

8. Suyama N, Mashimoto H, Araki J, Asai S, Ikeno Y, Ikeda T. An autopsy case of urinary bladder carcinoma with pulmonary infarction and subacute cor pulmonale caused by tumor embolization. Nihon Kyobu Shikkan Gakkai Zasshi (Japanese Journal of Thoracic Diseases) 32: 491-496, 1994 (in Japanese, Abstract in English).

9. Rüchardt A, Gruber B, Trenkwalder P. Intrapulmonary tumor cell embolism from cancer of the bladder as the cause of a subacute cor pulmonale. Dtsch Med Wochenschr 126: 847-850, 2001 (in German, Abstract in English).

10. Dhillon SS, Singh DJ, Dass B, Schaub CR. Transitional cell carcinoma manifesting as acute cor pulmonale: cause of microscopic tumor embolism. South Med J 94: 1030-1032, 2001.

11. Kobori G, Yamada H, Hirai S, Higashi Y. A case of microscopic pulmonary tumor emboli 14 months after total cystectomy. Hinyokika Kiyo (Acta Urologica Japonica) 50: 279-281, 2004 (in Japanese, Abstract in English).

12. Fujiwara H, Katsura H, Suemur M. A case of pulmonary tumor embolism presenting with an initial manifestation of urothelial carcinoma. Nihon Kokyuki Gakkai Zasshi (Journal of the Japanese Respiratory Society) 44: 504-510, 2006 (in Japanese, Abstract in English).

13. Chertcoff FJ, Emery NC, Villagomez R, et al. Pulmonary tumor embolism: report of two cases. Rev Med Chil 137: 1613-1616, 2009 (in Spanish, Abstract in English).

14. Hirano H, Ichibori H, Kizaki T, et al. Pulmonary tumor thrombotic microangiopathy showing aggressive course after transurethral resection of urinary bladder: an autopsy case report. Med Mol Morphol 45: 238-242, 2012.

15. Schriner RW, Ryu JH, Edwards WD. Microscopic pulmonary tumor embolism causing subacute cor pulmonale: a difficult antemortem diagnosis. Mayo Clin Proc 66: 143-148, 1991.

16. Janower ML, Blennerhassett JB. Lymphangitic spread of metastatic cancer to the lung. A radiologic-pathologic classification. Radiology 101: 267-273, 1971.

17. Veinot JP, Ford SE, Price RG. Subacute cor pulmonale due to tumor embolization. Arch Pathol Lab Med 116: 131-134, 1992.

18. Crane R, Rudd TG, Dail D. Tumor microembolism: pulmonary perfusion pattern. J Nucl Med 25: 877-880, 1984.

The Internal Medicine is an Open Access article distributed under the Creative Commons Attribution-NonCommercial-NoDerivatives 4.0 International License. To view the details of this license, please visit (https://creativecommons.org/licenses/ by-nc-nd/4.0/).

(C) 2016 The Japanese Society of Internal Medicine

http://www.naika.or.jp/imonline/index.html 